\title{
Evaluación de la percepción de un grupo universitario de docentes frente a la educación como fenómeno social en Colombia
}

\author{
Evaluation of the Perception of a University Group of Teachers about \\ Education as a Social Phenomenon in Colombia
}

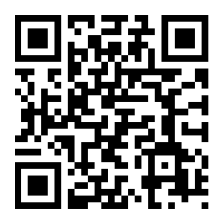

\author{
Jorge Enrique Moreno-Collazos ${ }^{1}$ \\ Universidad de la Sabana \\ Chia, Colombia \\ jorge.moreno2@unisabana.edu.co \\ Harold Fabián Cruz-Bermudez ${ }^{2}$ \\ Instituto del Corazón de Bucaramanga \\ Bogotá, Colombia \\ haroldcruzcx@gmail.com \\ Adriana Angarita-Fonseca ${ }^{3}$ \\ Universidad de Santander \\ Bucaramanga,Colombia \\ adriangarita@udes.edu.co \\ Amparo Vélez-Ramírez ${ }^{4}$ \\ Universidad Minuto de Dios \\ Bogotá, Colombia \\ amvelez@uniminuto.edu
}

Recibido 27 de junio de 2015 • Corregido 1 de julio de 2016 • Aceptado 16 de agosto de 2016

\footnotetext{
${ }^{1}$ Fisioterapeuta, director de grupo de Movimiento Corporal Humano Universidad de La Sabana, Especialista en Rehabilitación Cardiopulmonar, Magister en Ciencias, Doctor en Salud Pública, Doctorado en Educación (c) Posdoctorado en Educación, Ciencias Sociales e Interculturalidad (c). Estudiante Universidad de Baja California México. Estudiante Universidad de Santo Tomás.

2 Enfermero, especialista en Estadística Aplicada, Doctor en Ciencias de la Salud mención en Epidemiología, Instituto del Corazón de Bucaramanga, Doctorado en Educación (c) Posdoctorado en Educación, Ciencias Sociales e Interculturalidad (c). Estudiante Universidad de Baja California México. Estudiante Universidad de Santo Tomás.

${ }^{3}$ Fisioterapeuta, Magíster en Epidemiología y Estadística, docente e investigadora Universidad de Santander Universidad Santo Tomás, seccional Bucaramanga.

${ }^{4}$ Sociologa, directora de investigación Universidad Minuto de Dios, Especialista en Métodos y Técnicas Avanzadas en Investigación y Ciencias Sociales, Magister en Investigación y Docencia, Doctora en Filosofía. Posdoctorado en Educación, Ciencias Sociales e Interculturalidad (c). Estudiante Universidad de Santo Tomás.
} 
doi: http://dx.doi.org/10.15359/ree.20-3.8

URL: http://www.una.ac.cr/educare

CORREO: educare@una.cr

Resumen. El objetivo del presente artículo busca evaluar la percepción de un grupo universitario de docentes frente a la educación como fenómeno social. Se realizó un estudio de corte transversal analítico con un grupo de docentes de universidad de la ciudad de Bogotá en el periodo de octubre de 2014. Se aplicó una encuesta con el fin de evaluar la percepción frente a la educación como un fenómeno social; el análisis de los datos se realizó en el programa SPSS versión 19. La población de estudio estuvo conformada por 60 docentes de universidad con un promedio de edad de 42,2 \pm 14,4 años, un tiempo de ejercicio docente de 6,15 años; el género predominante fue masculino con un 53\% $(n=32)$. Se encontraron diferencias estadísticamente significativas para la dimensión de concepción de la educación ( $p=0,05-0,00)$, y los ítems “ ¿Considera que la inversión en educación pública es suficiente?" $(p=0,02)$, "¿La educación es un modelo político?" $(p=0,03)$; en edad agrupada "¿El acceso a la educación superior es concebido por el estado colombiano con un enfoque social?", “ ¿El entorno influye en el desarrollo educativo del estudiante colombiano?" $(p=0,02-0,00)$; igual ocurre para la sociología interpretativa para el ítem "¿Los patrones de valoración educativa son adecuados en el país?" $(p=0,04)$. Como conclusiones, se halló que existen diferencias entre las concepciones docentes sobre la construcción de la educación como fenómeno social.

Palabras claves. Educación, desarrollo, historia, investigación, sociología.

Abstract. Objective: This paper aims to evaluate the perception of a university group of teachers about education as a social phenomenon. An analytical cross-sectional study was conducted with a group of university teachers in Bogota, Colombia, in October 2014. A survey was applied in order to evaluate the perception about education as a social phenomenon; the data analysis was performed using SPSS software version 19. The population studied consisted of 60 university professors with an average age of $42.2 \pm 14.4$ years, and with 6.15 years of teaching practice. Male gender was predominant with $53 \%$ $(n=32)$. There were statistically significant differences in the results related to the concept of education $(p=0.05$ to 0.00$)$ and to the following items: "Do you consider that investment in public education is enough?" ( $p=0.02)$, "Is education a political model?" $(P=0.03)$; in aggregate age, "Is access to higher education conceived by the Colombian government with social concern?", "Does the environment influence the Colombian student's education development?" ( $p=0.02$ to 0.00$)$; it was the same case for the interpretive sociology for the item "Are patterns of educational assessment adequate in the country?" ( $p=0.04)$. In conclusion, it was found that there are differences in teachers' conceptions about the construction of education as a social phenomenon.f education as a social phenomenon by finding differences between the conceptions of it in teaching.

Keywords. Education, development, history, research, sociology.

\section{Introducción}

Las corrientes pedagógicas en la sociedad involucran, cada vez más, los aspectos relevantes de la sociedad, en donde, a lo largo de la existencia, la educación, desde su concepción histórica, ha desarrollado estrategias que permiten influenciar aspectos sociales en los que se desarrolla el estudiantado, por tal razón las disciplinas intelectuales se ocupan del ser humano como ser social 
y también por medio del método de la razón. Este tipo de interacciones metodológicas hacen que la participación en los aspectos involucrados en el desarrollo conceptual de la persona, como un universo y como miembro de la sociedad, interfieren sobre los grupos y las sociedades. De esto se han construido líneas de intervención que hacen que el conocimiento de las ciencias sociales de las ciencias físicas y biológicas se distinga (González-Moro y Caldero, 2010).

En cuanto a la relación de la sociología con el componente histórico de la educación como tal, se puede evidenciar que en este proceso se distinguen varios aspectos importantes. El primero orientado a los inicios y antecedentes de la sociología y su vínculo con los procesos investigativos abarcados en los años 60, lugar del tiempo en donde los aportes de investigación y educación forjaron los primeros pilares fundamentales de la educación como un proceso evolutivo. El segundo momento se orientó hacia la influencia de las investigaciones de la sociología en los años de 1960 a 1970, en las que encontramos directrices importantes en la construcción de modelos sociológicos en la educación de la actualidad. Por último, un tercer momento en donde las corrientes sociológicas han permitido la evolución de la educación hasta hoy (Bartolomé, 2004).

Enmarcando la evolución de la idea sociológica en el entorno colombiano, es necesario remontarse a su nacimiento, que de la misma manera como aparecieron todas las ciencias sociales en este país, vivió un largo y arduo proceso para su consolidación, contrayéndose y posicionándose como una ciencia hoy. La universidad comenzó a interesarse en la formación de personal para la investigación en esta área hacia la década de los 80 , con la conformación de la Escuela Normal Superior y en el Instituto Etnológico Nacional (Cataño, 1987).

El último fenómeno plantea las relaciones entre la sociología y el Estado; estas relaciones son de carácter político y económico (Sánchez, 2006). En las relaciones políticas se han dado contrastes que van desde la colaboración hasta la oposición. En el ámbito económico, se hace referencia a la"participación de la sociología como ciencia y como base del concepto y la práctica de la planeación en el proceso de desarrollo de la sociedad, como a la apertura de posibilidades ocupacionales para los profesionales de la sociología" (Parra, 1989, p. 68).

Colombia ha tenido un largo recorrido, influenciado por varios factores, para desarrollar la investigación relacionada con la interrelación de la sociedad y la educación como modelo interactivo entre el entorno del ser humano, en donde la sociología ha avanzado significativamente. Actualmente existen, por ejemplo, programas de postgrado orientados al estudio de la dinámica social en nuestro país, con miras a la organización y planificación del sistema educativo; todos estos esfuerzos han permitido que la calidad de la educación sea un reto constante para las instituciones educativas (Ocampo, 2009). 
doi: http://dx.doi.org/10.15359/ree.20-3.8

URL: http://www.una.ac.cr/educare

CORREO: educare@una.cr

En el caso particular de Colombia, es necesario realizar un acercamiento más profundo que propenda por identificar qué valoración se hace hoy de la investigación; cómo se articula con los procesos de generación de programas de educación superior $y$, si estos proyectos curriculares contemplan la sociología y la educación como un pilar fundamental en el desarrollo profesional de la población y cómo esta aporta significativamente al desarrollo del país (Bauman, 2002).

Por lo anterior, el objetivo de este estudio fue evaluar la percepción de un grupo universitario de docentes frente a la educación como fenómeno social.

\section{Método}

Se realizó un estudio de corte transversal analítico con un grupo de universitario de docentes $(n=60)$ de la ciudad de Bogotá, en el periodo de octubre de 2014. Para la recolección de la información se construyó una encuesta no estructurada con el fin de evaluar la percepción docente frente al texto La sociedad y educación: Desde una perspectiva sociológica (el cual se pude consultar por medio del buscador Google), con el fin de indagar la percepción de la educación como un fenómeno social y conocer la posición frente a esta misma. Inicialmente se entregó a cada participante el documento para su lectura y, posteriormente, se aplicó un instrumento tipo encuesta. La obtención de la muestra fue no probabilística con la metodología bola de nieve. La encuesta fue anónima y se guardó la confidencialidad de la institución universitaria correspondiente.

\section{Instrumento}

Con el fin de validar los datos y evitar sesgos, la encuesta fue sometida a evaluación por un grupo de 10 personas expertas en el área de educación, quienes calificaron las preguntas con los siguientes valores de pertinencia: $1=$ no pertinente, $2=$ poco pertinente, $3=$ pertinente y $4=$ muy pertinente. Para la operacionalización de los datos y dar validez al proceso, se estableció que el valor mínimo para la validación era de 10 personas expertas en educación y pedagogía; para la validación de contenido del instrumento se debió obtener como mínimo una puntuación de 0,62. Para el cálculo se utilizó la formula índice de validez de contenido IVC = número de ítems con puntuación entre 3 y 4 / número total de ítems. Esta fórmula se aplicó para cada calificación dada por el personal experto. Luego se aplicó la formula índice de validez de contenido general IVCG = suma del IVC calculado para cada persona experta / número de personas expertas. Para realizar la evaluación de la consistencia interna se aplicó el estadístico alfa de Cronbach, el cual es un coeficiente que sirve para medir la fiabilidad de una escala, con una medida estimada de obtención por encima de 0,80 . Las dimensiones del instrumento evaluaron 4 subtemas que busca un acercamiento a la sociología de la educación, las respuestas son dicotómicas Sí/NO (Ver tabla 1).

4

Jorge Enrique Moreno-Collazos, Harold Fabián Cruz-Bermudez, Adriana Angarita-Fonseca y Amparo Vélez-Ramírez 
Tabla 1

Distribución de constructo evaluado en la encuesta

\begin{tabular}{|c|c|}
\hline Dimensión & Ítem de la encuesta \\
\hline \multirow[t]{2}{*}{ Educación } & $\begin{array}{l}\text { 1. ¿Considera usted que la educación colombiana se relaciona con el } \\
\text { comportamiento social del país? }\end{array}$ \\
\hline & 2. ¿Los programas educativos contemplan la dinámica social colombiana? \\
\hline \multirow{2}{*}{ Sociología de la educación } & $\begin{array}{l}\text { 3. ¿La educación tiene influencia en el desarrollo económico del futuro sujeto } \\
\text { profesional? }\end{array}$ \\
\hline & 4. Considera que la inversión económica en educación pública es suficiente? \\
\hline \multirow{3}{*}{ Enfoques educativos } & $\begin{array}{l}\text { 5. ¿El acceso a la educación superior es concebido por el Estado colombiano con } \\
\text { un enfoque social? }\end{array}$ \\
\hline & 6. ¿La educación es un modelo político? \\
\hline & 7. ¿El entorno influye en el desarrollo educativo del estudiantado colombiano? \\
\hline \multirow{3}{*}{ Sociología interpretativa } & $\begin{array}{l}\text { 8. ¿La educación debe construirse desde un modelo cambiante según el cambio } \\
\text { social? }\end{array}$ \\
\hline & 9. ¿El sistema curricular colombiano está basado en los estilos pedagógicos? \\
\hline & 10. ¿Los patrones de valoración educativa son adecuados al país? \\
\hline
\end{tabular}

Nota: Elaboración propia.

\section{Análisis estadístico}

Se aplicaron medidas de tendencia central y de dispersión para las variables cuantitativas con sus respectivos intervalos de confianza del 95\% (IC 95\%) y frecuencias absolutas y relativas para variables cualitativas; las variables edad y tiempo (años) de ejercicio docente se agruparon tomando como punto de corte la mediana. En el análisis bivariado se aplicó una prueba de contraste de interdependencia chi-cuadrado con el fin de establecer diferencias o similitudes entre los ítems evaluados según género, edad y ejercicio docente, para el caso de frecuencias esperadas $<5$ se utilizó el estadístico test exacto de Fisher. Para todo el análisis se utilizó un alpha de 0.05 . El análisis de los datos se realizó en el programa estadístico SPSS 19 de IBM ${ }^{\oplus}$.

\section{Consideraciones éticas}

La totalidad de participantes firmó el consentimiento informado, que tiene en cuenta los aspectos éticos institucionales para la investigación. Se mantuvo la confidencialidad del personal docente en relación con su filiación institucional. 
doi: http://dx.doi.org/10.15359/ree.20-3.8

URL: http://www.una.ac.cr/educare

CORREO: educare@una.cr

\section{Resultados}

La población de estudio estuvo conformada por 60 docentes de universidades de la ciudad de Bogotá con un promedio de edad de 42,2 \pm 14,4 (IC 95\% 38,54-15,99) años, un tiempo de ejercicio docente de 6,15 $\pm 3,86$ (IC 95\% 5,15-7,15) años; el género predominante fue masculino con un $53 \%(n=32)$, como se observa en la tabla 2.

Tabla 2

Estadísticos descriptivos de la población de estudio

\begin{tabular}{|c|c|c|c|}
\hline Variables & & $n$ & $\%$ \\
\hline \multirow{2}{*}{ Género } & Masculino & 32 & $53 \%$ \\
\hline & Femenino & 28 & $47 \%$ \\
\hline \multirow{12}{*}{ Tiempo de ejercicio docente (años) } & 2 & 18 & $30 \%$ \\
\hline & 3 & 3 & $5 \%$ \\
\hline & 4 & 1 & $2 \%$ \\
\hline & 5 & 6 & $10 \%$ \\
\hline & 6 & 6 & $10 \%$ \\
\hline & 7 & 4 & $7 \%$ \\
\hline & 8 & 8 & $13 \%$ \\
\hline & 9 & 5 & $8 \%$ \\
\hline & 10 & 2 & $3 \%$ \\
\hline & 12 & 2 & $3 \%$ \\
\hline & 14 & 2 & $3 \%$ \\
\hline & 15 & 3 & $5 \%$ \\
\hline \multirow{2}{*}{ Edad (agrupada) } & $<=38$ & 31 & $52 \%$ \\
\hline & $39+$ & 29 & $48 \%$ \\
\hline \multirow{2}{*}{ Tiempo de años docencia (agrupada) } & $\leq 6$ años & 34 & $57 \%$ \\
\hline & $>6$ años & 26 & $43 \%$ \\
\hline
\end{tabular}

En el análisis bivariado, al comparar los ítems evaluados frente a género, edad y tiempo de ejercicio docente, se evidencian diferencias estadísticamente significativas en la dimensión de concepción de la educación ( $p=0,05-0,00)$, por otra parte, la sociología de la educación solamente se encuentran diferencia en el ítem "Considera que la inversión en educación pública es suficiente" $(p=0,02)$. 
Enladimensión enfoques educativos seencuentran diferenciasestadísticamente significativas por género para el ítem "¿La educación es un modelo político?" $(p=0,03)$, en edad agrupada "¿El acceso a la educación superior es concebido por el Estado colombiano con un enfoque social" $y$ " $i E l$ entorno influye en el desarrollo educativo del estudiantado Colombiano?" $(p=0,02-0,00)$; lgual ocurre para la sociología interpretativa para el ítem "¿Los patrones de valoración educativa son adecuados en el país?" $(p=0,04)$. Los datos correspondientes se muestran en la tabla 3 .

Tabla 3

Análisis bivariado de respuesta del instrumento de evaluación frente a género, edad agrupada y tiempo de ejercicio docente agrupado

\begin{tabular}{|c|c|c|c|c|c|c|c|c|c|c|c|c|c|c|c|c|}
\hline \multirow{3}{*}{\multicolumn{2}{|c|}{ Ítem evaluado }} & \multicolumn{4}{|c|}{ Género } & \multirow{3}{*}{$p^{*}$} & \multicolumn{4}{|c|}{ Edad (agrupado) } & \multirow{3}{*}{$p^{*}$} & \multicolumn{4}{|c|}{ Tiempo de ejercicio docente (agrupado) } & \multirow{3}{*}{$p^{*}$} \\
\hline & & \multicolumn{2}{|c|}{ Masculino } & \multicolumn{2}{|c|}{ Femenino } & & \multicolumn{2}{|c|}{$<=38$} & \multicolumn{2}{|c|}{$39+$} & & \multicolumn{2}{|c|}{$\leq 6$ años } & \multicolumn{2}{|c|}{$>6$ años } & \\
\hline & & $\mathrm{n}$ & $\%$ & $\mathrm{n}$ & $\%$ & & $\mathrm{n}$ & $\%$ & $\mathrm{n}$ & $\%$ & & $\mathrm{n}$ & $\%$ & $\mathrm{n}$ & $\%$ & \\
\hline \multirow[t]{2}{*}{1} & Sí & 15 & $25 \%$ & 14 & $23 \%$ & \multirow{2}{*}{0,05} & 19 & $32 \%$ & 10 & $17 \%$ & \multirow{2}{*}{4,31} & 19 & $32 \%$ & 10 & $17 \%$ & \multirow{2}{*}{1,79} \\
\hline & NO & 17 & $28 \%$ & 14 & $23 \%$ & & 12 & $20 \%$ & 19 & $32 \%$ & & 15 & $25 \%$ & 16 & $27 \%$ & \\
\hline \multirow[t]{2}{*}{2} & sí & 16 & $27 \%$ & 14 & $23 \%$ & \multirow{2}{*}{0,00} & 14 & $23 \%$ & 16 & $27 \%$ & \multirow{2}{*}{0,60} & 15 & $25 \%$ & 15 & $25 \%$ & \multirow{2}{*}{1,08} \\
\hline & NO & 16 & $27 \%$ & 14 & $23 \%$ & & 17 & $28 \%$ & 13 & $22 \%$ & & 19 & $32 \%$ & 11 & $18 \%$ & \\
\hline \multirow[t]{2}{*}{3} & Sí & 27 & $45 \%$ & 26 & $43 \%$ & \multirow{2}{*}{1,04} & 27 & $45 \%$ & 26 & $43 \%$ & \multirow{2}{*}{0,95} & 29 & $48 \%$ & 24 & $40 \%$ & \multirow{2}{*}{0,70} \\
\hline & NO & 5 & $8 \%$ & 2 & $3 \%$ & & 4 & $7 \%$ & 3 & $5 \%$ & & 5 & $8 \%$ & 2 & $3 \%$ & \\
\hline \multirow[t]{2}{*}{4} & SÍ & 12 & $20 \%$ & 10 & $17 \%$ & \multirow{2}{*}{0,02} & 10 & $17 \%$ & 12 & $20 \%$ & \multirow{2}{*}{0,53} & 14 & $23 \%$ & 8 & $13 \%$ & \multirow{2}{*}{0,68} \\
\hline & NO & 20 & $33 \%$ & 18 & $30 \%$ & & 21 & $35 \%$ & 17 & $28 \%$ & & 20 & $33 \%$ & 18 & $30 \%$ & \\
\hline \multirow[t]{2}{*}{5} & sí & 10 & $17 \%$ & 8 & $13 \%$ & & 9 & $15 \%$ & 9 & $15 \%$ & & 8 & $13 \%$ & 10 & $17 \%$ & \\
\hline & NO & 22 & $37 \%$ & 20 & $33 \%$ & נוכוט & 22 & $37 \%$ & 20 & $33 \%$ & $0,0 L$ & 26 & $43 \%$ & 16 & $27 \%$ & 1, \\
\hline 6 & Sí & 29 & $48 \%$ & 25 & $42 \%$ & & 27 & $45 \%$ & 27 & $45 \%$ & & 30 & $50 \%$ & 24 & $40 \%$ & \\
\hline & NO & 3 & $5 \%$ & 3 & $5 \%$ & $0,0 J$ & 4 & $7 \%$ & 2 & $3 \%$ & 0,00 & 4 & $7 \%$ & 2 & $3 \%$ & 0,27 \\
\hline 7 & sí & 27 & $45 \%$ & 27 & $45 \%$ & & 28 & $47 \%$ & 26 & $43 \%$ & & 32 & $53 \%$ & 22 & $37 \%$ & \\
\hline & NO & 5 & $8 \%$ & 1 & $2 \%$ & $2,+1$ & 3 & $5 \%$ & 3 & $5 \%$ & 0,00 & 2 & $3 \%$ & 4 & $7 \%$ & 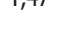 \\
\hline 8 & SÍ & 31 & $52 \%$ & 23 & $38 \%$ & & 25 & $42 \%$ & 29 & $48 \%$ & & 29 & $48 \%$ & 25 & $42 \%$ & \\
\hline & NO & 1 & $2 \%$ & 5 & $8 \%$ & & 6 & $10 \%$ & 0 & $0 \%$ & & 5 & $8 \%$ & 1 & $2 \%$ & נכ, \\
\hline 9 & Sí & 32 & $53 \%$ & 28 & $47 \%$ & $* *$ & 31 & $52 \%$ & 29 & $48 \%$ & $* *$ & 34 & $57 \%$ & 26 & $43 \%$ & $* *$ \\
\hline 10 & SÍ & 26 & $43 \%$ & 21 & $35 \%$ & & 21 & $35 \%$ & 26 & $43 \%$ & & 25 & $42 \%$ & 22 & $37 \%$ & \\
\hline & NO & 6 & $10 \%$ & 7 & $12 \%$ & 0,34 & 10 & $17 \%$ & 3 & $5 \%$ & 0,04 & 9 & $15 \%$ & 4 & $7 \%$ & Lל \\
\hline
\end{tabular}

Nota: * Prueba chi-cuadrado o test exacto de Fisher; significativo $<0,05^{* *}$ No se realiza prueba por constante. Elaboración propia. 
doi: http://dx.doi.org/10.15359/ree.20-3.8

URL: http://www.una.ac.cr/educare

CORREO: educare@una.cr

\section{Discusión}

El propósito general de la investigación fue analizar la percepción que tienen los grupos docentes universitarios frente a la educación como un modelo social y, adicionalmente, conocer si existían diferencias entre la construcción de conocimientos frente a la situación que enfrenta nuestro país en relación con los modelos educativos (Díaz-Barriga, 1993). La percepción docente en los modelos educativos se convierte en factor determinante al momento de definir políticas educativas; el tiempo de ejercicio docente como algunos factores relacionados con la experiencia del ejercicio profesional hacen que la evaluación de la educación en un contexto social varíe, como se pudo evidencia en el estudio, en donde se evidencian diferencias estadísticamente significativas en la dimensión de concepción de la educación (Cordié, 1998).

En la actualidad se encuentran, cada vez más, docentes jóvenes que se han formado después de los cambios educativos, por lo cual se espera que la sociología de la educación tenga mayor influencia en su formación (Subirats y Nogales, 1989).

La inversión en educación en Colombia responde "a una necesidad social de recuperar la experiencia acumulada y decantada en la tradición" (Misas, 2004, p. 13), en donde los costos destinados para esta parten de unas características económicas del entorno y el desarrollo de la población, adicionalmente los entes gubernamentales mitigan esfuerzos para hacer que los recursos sean distribuidos, según la evaluación previa de las necesidades educativas. De igual manera, para el caso de los hallazgos del estudio, el personal docente con diferencias entre sus opiniones, consideran que la educación es un modelo político. La educación permite, a las nuevas generaciones de una sociedad, reconocer los símbolos que identifican esa sociedad: la inversión monetaria hace que los individuos inmersos dentro de una sociedad desarrollen habilidades que fortalecen el desarrollo de un territorio (Misas, 2004).

Los acercamientos entre sociedad, docente y universidad se suceden de diferentes formas, por tal razón las oportunidades se desarrollan en procesos que no necesariamente involucran los proyectos curriculares; las universidades contemplan el acceso estudiantil con diversas razones: ya sean relacionadas con las políticas del Estado (como los recortes presupuestales y la necesidad de obtener recursos para financiar su funcionamiento), con la naturaleza misma de la institución, que privilegia algún sector económico o social en particular. Esta orientación se encuentra se evaluó en los sujetos participantes del estudio con la pregunta "¿El entorno influye en el desarrollo educativo del estudiantado colombiano"? Es indispensable que ahora los diversos entes evaluadores de la situación educativa colombiana actual puedan hablar de una perspectiva social.

En relación con el acceso de la educación, se encontró que como en todos los países existen procesos de evaluación económica, social y relacionada con conocimientos previos adquiridos (Moloney, 2010), en Colombia también: los procesos de selección para ingreso a la

8

Jorge Enrique Moreno-Collazos, Harold Fabián Cruz-Bermudez, Adriana Angarita-Fonseca y Amparo Vélez-Ramírez 
educación superior se basan en características que le permitirán al estudiantado desarrollar su potencia como profesional en el futuro, frente a esto, el personal docente evaluado difiere de las políticas de ingreso a la educación (Sabelli, 2003).

La sociología interpretativa aplicada al modelo educativo colombiano realiza aproximaciones a los modelos investigativos en los cuales las necesidades educativas se adaptan a un entorno social (Chacín, González y Torres, 2007), en donde se establece en la actualidad en nuestro país, la evaluación de logros que le permiten al individuo desarrollar competencias concordantes con las mismas habilidades personales. Los modelos de calificación y cuantificación de los saberes deben integrar este tipo de aspectos, el profesorado colombiano continúa pensando que existe la necesidad de evaluar los sistemas métricos de evaluación, aunque hay diferencia en lo que desean evaluar (Pérez, Peña y Torres, 2011).

En relación con la trascendencia del ejercicio profesional, los años de docencia demarcan que una trayectoria académica en el aula permita una construcción teórica robusta crítica y reflexiva, independientemente del género. Al respecto, se introyecta un mayor compromiso social con las necesidades de la sociedad, cuando los educadores y educadoras se conciben como agentes de transformación cultural y tradiciones que fomentan una transformación de acuerdo con las exigencias sociales (Zúñiga, 2015).

\section{Conclusiones}

En Colombia es necesario seguir realizando tipos de investigaciones reflexivas en relación con la sociología de la educación, a pesar de los procesos que se pueden suscitar en las problemáticas sociales y políticas en las que se ven enfrentados, día tras día, los sistemas educativos; pues este es el camino que nos puede llevar a generar líneas fuertes en investigación social, para generar avances en la investigación aplicada en las ciencias de la educación en cuanto a la cultura social.

\section{Agradecimientos}

A las directivas y docentes de las instituciones universitarias de la ciudad de Bogotá que participaron en el diligenciamiento del cuestionario de la investigación.

\section{Conflictos de intereses}

No existen conflictos de interés al tratarse de un estudio en donde se guardó la confidencialidad de las instituciones a las que pertenecía cada docente. 
doi: http://dx.doi.org/10.15359/ree.20-3.8

URL: http://www.una.ac.cr/educare

CORREO: educare@una.cr

\section{Referencias}

Bartolomé, A. (Abril, 2004). Blended learning. Conceptos básicos. Pixel Bit Revista de Medios y Educación, 23, 7-20. Recuperado de http://www.sav.us.es/pixelbit/pixelbit/articulos/n23/ PIXEL BIT 23.pdf

Bauman, Z. (2002). La cultura como praxis. Barcelona: Paidós.

Cataño, G. (1987). La sociología en Colombia. Bogotá: Plaza \& Janes.

Chacín, B., González, M. y Torres, Y. (2007). Crítica a la generación del conocimiento en la extensión universitaria: Aproximación a un protocolo de investigación innovativa. Laurus, 13(24), 215-240. Recuperado de http://www.redalyc.org/articulo.oa?id=76111485011

Cordié, A. (1998). Malestar en el docente. La educación confrontada con el psicoanálisis. Buenos Aires: Nueva Visión.

Díaz-Barriga, A. (1993). 4. Los procesos de frustración en la tarea docente. En Á. Díaz-Barriga (Aut.), Tarea docente. Una perspectiva didáctica grupal y psicosocial (pp. 73-99). México: Nueva Imagen.

González-Moro, M. E. y Caldero, J. (2010). Didáctica de las ciencias sociales. Salamanca: Universidad de Salamanca.

Misas, G. M. (2004). La educación superior en Colombia. Análisis y estrategias para su desarrollo. Bogotá: Universidad Nacional de Colombia. Recuperado de http://www.colombiaaprende. edu.co/html/investigadores/1609/articles-73081 archivo.pdf

Moloney, C. (Abril, 2010). Understanding Understanding Across the Disciplines: Towards Strategies for Sustainable Engineering Education for the 21st Century [Entendiendo el entendimiento en las disciplinas:Acercarse a estrategias para la educación en Ingeniería Sostenible en el siglo XXI]. En Transforming Engineering Education:Creating Interdisciplinary Skills for Complex Global Environments [Transformando la eduación en Ingeniería: Crear hablidades interdisciplinarias para ambientes globales complejos] (pp. 1-31). Dublín: IEEE. doi: http://dx.doi.org/10.1109/TEE.2010.5508815

Ocampo, L. J. (2009). El maestro Orlando Fals Borda sus ideas educativas y sociales para el cambio en la sociedad colombiana. Revista Historia de la Educación Latinoamericana, 12, 13-41. Recuperado de http://www.redalyc.org/articulo.oa?id=86912021002

Parra, R. (1989). La sociología en Colombia. Bogotá: Instituto Caro y Cuervo. 
Pérez, P. A., Peña, J. I. y Torres, E. (2011). A self-evaluation model as a tool for the evaluation of graduate programs at Universidad Nacional de Colombia [Un modelo de autoevaluación como herramienta de la evaluación de programas de posgrado en la Universidad Nacional de Colombia]. En EDULEARN11 Proceedings, 3rd International Conference on Education and New Learning Techonologies [3 ${ }^{a}$ Conferencia internacional de educación y nuevas tecnologías de aprendizaje] (pp. 661-672). Barcelona: IATED.

Sabelli, M. (Mayo-agosto, 2003). La enseñanza universitaria y su extensión en comunidades de frontera: Un proyecto dirigido a promover políticas sociales para el ciudadano como usuario de información y lectura. Transinformação, 15(2), 119-133. Recuperado de http:// periodicos.puc-campinas.edu.br/seer/index.php/transinfo/article/view/1481/1455

Sánchez, A. (Octubre, 2006). Ciencia, revolución y creencia en Camilo Torres: ¿Una Colombia secular? Revista Nómadas, 25, 241-258. Recuperado de http://www.redalyc.org/articulo. oa?id=105115224020

Subirats, J. y Nogales, I. (1989). Maestros, escuelas, crisis educativa. Condiciones del trabajo docente en Bolivia. Santiago, Chile: UNESCO-OREALC. Recuperado de http://unesdoc.unesco.org/ images/0008/000853/085394so.pdf

Zúñiga, W. (Enero-junio, 2015). Una perspectiva acerca de la investigación y la docencia universitaria en Colombia. EN-Clave Social, 4(1), 10-23. Recuperado de http:// http:// repository.lasallista.edu.co:8080/ojs/index.php/EN-Clave/article/view/947/674

\section{Cómo citar este artículo en APA:}

Moreno-Collazos, J. E., Cruz-Bermudez, H. F., Angarita-Fonseca, A. y Vélez-Ramírez, A. (Setiembrediciembre, 2016). Evaluación de la percepción de un grupo universitario de docentes frente a la educación como fenómeno social en Colombia. Revista Electrónica Educare, 20(3), 1-11. doi: http://dx.doi.org/10.15359/ree.20-3.8

Nota: Para citar este artículo en otros sistemas puede consultar el hipervínculo "Como citar el artículo" en la barra derecha de nuestro sitio web: http://www.revistas.una.ac.cr/index.php/EDUCARE/index 